Note: This is a preprint of a paper submitted for publication. Contents of this paper should not be quoted or referred to without permission of the author(s).

$$
\text { CONF-960163--21 }
$$

Submitted for publication in:

Oxide Superconductor Physics and Nano-Engineering II

SPIE Conference Proceedings Vol. 2697

Editors: D. Pavuna and I. Bozovic

SPIE, Bellingham, WA (1996)

\title{
DEFECT FORMATION AND CARRIER DOPING IN EPITAXIAL FILMS OF THE INFINITE LAYER COMPOUND
}

\author{
R. Feenstra, S. J. Pennycook, M. F. Chisholm, N. D. Browning, \\ J. D. Budai, D. P. Norton, E. C. Jones, D. K. Christen \\ Solid State Division, Oak Ridge National Laboratory \\ P.O. Box 2008, Oak Ridge, Tennessee 37831-6057
}

T. Matsumoto and T. Kawai

Institute of Scientific and Industrial Research, Osaka University

8-1 Mihogaoka, Ibaraki, 567 Osaka, Japan

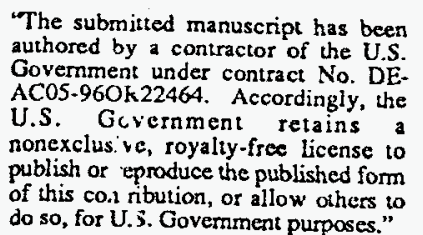

authored by a manuscript has been

Government under contract No. DE.

AC05-960k 22464. Accordingly, the

U.S. Guvernment retains

publish or eproduce to

dhis coi ribution, or allow others

Prepared by the

SOLID STATE DIVISION

OAK RIDGE NATIONAL LABORATORY

Oak Ridge, Tennessee 37831-6061

Managed by

LOCKHEED MARTIN ENERGY RESEARCH CORP.

for the

U.S. DEPARTMENT OF ENERGY

under

Contract No. DE-AC05-96OR22464

February 1996 


\section{DISCLAIMER}

This report was prepared as an account of work sponsored by an agency of the United States Government. Neither the United States Government nor any agency thereof, nor any of their employees, makes any warranty, express or implied, or assumes any legal liability or responsibility for the accuracy, completeness, or usefulness of any information, apparatus, product, or process disciosed, or represents that its use would not infringe privately owned rights. Reference herein to any specific commercial product, process, or service by trade name, trademark, manufacturer, or otherwise does not necessarily constitute or imply its endorsement, recommendation, or favoring by the United States Government or any agency thereof. The views and opinions of authors expressed herein do not necessarily state or reflect those of the United States Government or any agency thereof. 


\section{DISCLAIMER}

Portions of this document may be illegible in electronic image products. Images are produced from the best available original document. 


\title{
Defect formation and carrier doping in epitaxial films of the infinite layer compound
}

\author{
R. Feenstra, S. J. Pennycook, M. F. Chisholm, N. D. Browning, \\ J. D. Budai, D. P. Norton, E. C. Jones, and D. K. Christen \\ Solid State Division, Oak Ridge National Laboratory, \\ Oak Ridge, Tennessee 37831-6057
}

\author{
T. Matsumoto and T. Kawai \\ Institute of Scientific and Industrial Research, \\ Osaka University, Osaka, 567 Japan
}

\begin{abstract}
The correlation between defect formation and carrier doping in epitaxial films of the infinite layer compound $\mathrm{SrCuO}_{2}$ has been studied via molecular beam epitaxy controlled layer-by-layer growth experiments, chemically resolved scanning transmission electron microscopy, scanning tunneling microscopy, $\mathrm{x}$-ray diffraction, electrical transport measurements, and post-growth oxidation-reduction annealing. Based on the complementary information provided by these experiments, it is concluded that the carrier doping is dominated by the formation of an electrondoped, $\mathrm{Sr}$ and $\mathrm{O}$ deficient matrix under mildly oxidizing growth conditions. Hole-doping is induced by extended defects containing excess $\mathrm{Sr}$ atoms and may lead to superconductivity after high-temperature oxidation.
\end{abstract}

keywords: cuprate superconductors, infinite layer compound, atomic layer epitaxy, electron microscopy, scanning tunneling microscopy, oxidation-reduction annealing

\section{INTRODUCTION}

Following the high-pressure stabilization of Sr-rich solid solutions ${ }^{1}$ of the infinite layer (IL) compound $A \mathrm{CuO}_{2}$ $(A: \mathrm{Ca}-\mathrm{Sr}-\mathrm{Ba})$ and the observation of electron-doped and hole-doped superconductivity ${ }^{2,3}$ after appropriate doping, several new superconductors with $T_{c}>100 \mathrm{~K}$ have been synthesized using similar techniques. ${ }^{4}$ The IL compound represents the fundamental copper-oxygen/cation building block of the high- $T_{c}$ superconducting cuprates and provides the ultimate starting point for a systematic search of new materials. The role of the high-pressure synthesis conditions has been attributed to the different compressibilities of the $\mathrm{Cu}-\mathrm{O}$ and $A-\mathrm{O}$ bonds, ${ }^{1,5}$ affecting the lattice matching between constituent lattice planes and, consequently, the thermodynamics of phase formation. It has been suggested that a similar pressure effect may emanate from epitaxial strain and thus provide a driving force for formation of the IL compound on a lattice matched substrate. ${ }^{6-9}$ Indeed, IL films with compositions spanning the entire Ca-Sr-Ba solid solution ranges ${ }^{10,11}$ have been epitaxially grown on (100) $\mathrm{SrTiO}_{3}$ substrates by various authors, using low growth temperatures $\left(500-600^{\circ} \mathrm{C}\right.$ ) where the IL phase apparently becomes "trapped" because of reduced atomic mobilities. It is of basic as well as practical interest to utilize these IL films for the deposition controlled synthesis of new high- $T_{c}$ compounds, and investigate the properties of these highly oriented deposits.

During the past four years we have studied the synthesis of epitaxial films of the solid solution endmember $\mathrm{SrCuO}_{2}$ and characterized its properties. ${ }^{12,13} \mathrm{SrCuO}_{2}$ is an interesting member of the IL family for several reasons. First, because of the intermediate size of $\mathrm{Sr}^{2+}$ ions, tetragonal $\mathrm{SrCuO}_{2}$ resembles the original "all-layer" compound ${ }^{14}$ $\mathrm{Ca}_{0.86} \mathrm{Sr}_{0.14} \mathrm{CuO}_{2}$ which, in turn, is isomorphic with the $\mathrm{Ca}_{n-1} \mathrm{Cu}_{\mathrm{n}} \mathrm{O}_{2 \mathrm{n}}$ plane groups of high- $T_{c}$ homologous series in the limit $\mathrm{n} \rightarrow \infty$. Ideally, this structure consists of flat $\mathrm{CuO}_{2}$ sheets separated by $\mathrm{Sr}^{2+}\left(\mathrm{Ca}^{2+}\right)$ ions only. This structure is essentially undoped. On the other hand, $\mathrm{Sr}$ is large enough to allow for the interface between a $\mathrm{SrO}$ layer and a $\mathrm{CuO}_{2}$ plane (as found in the Bi-based cuprates, for example), introducing apical oxygen atoms in the structure and the possibility of "modulation doping" via the insertion of charge reservoir layers. This latter configuration is found exclusively for $\mathrm{Ba}$ in the high- $T_{c}$ cuprates. Secondly, $\mathrm{SrCuO}_{2}$ constitutes the parent of the electron-doped superconductors ${ }^{3,15} \mathrm{Sr}_{1-\mathrm{x}} R_{\mathrm{x}} \mathrm{CuO}_{2}(R=\mathrm{La}, \mathrm{Nd}, .$.$) with T_{c} \simeq 40 \mathrm{~K}$ for $\mathrm{x} \simeq 0.1$. These IL compounds represent the simplest cuprate superconductors possible and, therefore, are of interest for basic studies towards the 
origin of (electron-doped) superconductivity. Alternatively, $\mathrm{SrCuO}_{2}$ may be used as a "building block" for holedoped superconductors in superlattice structures with $\left[\mathrm{BaCuO}_{\mathrm{y}}\right]_{\mathrm{m}}(\mathrm{m}=2,3)$ charge reservoir layers. ${ }^{16}$ Because of the somewhat larger lattice parameters of $\mathrm{SrCuO}_{2}$ compared to $\mathrm{CaCuO}_{2}$, the lattice mismatch with $\mathrm{BaCuO}_{\mathrm{y}}$ is reduced, making it possible to better control the hetero-epitaxial growth process.

In this paper, we present results from electrical transport measurements, annealing studies to control the oxygen stoichiometry, chemically resolved scanning transmission electron microscopy (Z-contrast STEM), and ultra high vacuum scanning tunneling microscopy (UHV-STM) to characterize the films. Furthermore, atomic layer epitaxy (ALE) growth experiments were performed to synthesize the IL structure and modify its defect structure. This was achieved by hetero-epitaxial growth of $\operatorname{Sr}(\mathrm{O})$ and $\mathrm{CuO}$ monolayers in a laser ablation molecular beam epitaxy (laserMBE) system, in situ monitored by reflection high energy electron diffraction (RHEED). It is shown that the sign of the majority charge carriers changes from n-type (electron-doped) to p-type (hole-doped) when SrO defects are inserted in the IL lattice. A model is formulated that correlates the defect formation and carrier doping in IL films.

\section{EXPERIMENTAL}

$\mathrm{Sr}_{\mathrm{z}} \mathrm{CuO}_{2} \pm \delta$ films with various $\mathrm{z}=\mathrm{Sr} / \mathrm{Cu}$ compositions were epitaxially grown on (100) $\mathrm{SrTiO}_{3}$ substrates either by codeposition via single target pulsed laser ablation, or atomic layer epitaxy using laser-MBE. Details of the laser ablation system at ORNL and the laser-MBE system at Osaka University have been described previously. ${ }^{9,12}$ The film growth at ORNL was performed in a pure oxygen ambient at pressures between 2-200 mTorr. With the laserMBE, film growth took place in a flux of $\mathrm{NO}_{2}$ at background pressures of $0.3-4 \cdot 10^{-5} \mathrm{mbar}$, sufficiently low for continuous operation of the RHEED. Composite $\mathrm{SrCuO}_{2}$ targets, as well as metallic $\mathrm{Sr}$ and sintered $\mathrm{CuO}$ targets were used, depending on the mode of operation. Substrate temperatures in both systems were kept at $500-550^{\circ} \mathrm{C}$. In the laser-MBE experiments, a $\mathrm{SrO}$ monolayer was deposited first on the bare substrate to change the lattice termination from predominantly $\mathrm{TiO}_{2}$ to $\mathrm{SrO}$. In previous studies it was observed that this substrate pretreatment enhances the epitaxial growth mechanism of the IL films and is essential for initiating the atomic layer-by-layer growth scheme. ${ }^{6}$ The preparation of a proper substrate termination and its effect on the epitaxial growth process recently have been described by Kawasaki et al. ${ }^{17}$

Films grown with either technique were essentially single phase by $\mathrm{x}$-ray diffraction (XRD) and epitaxial with the $\mathrm{SrTiO}_{3}$ substrate. The orientation was such that the IL $c$-axis was perpendicular to the substrate surface and the tetragonal $a$-axes parallel to the in-plane [100] directions. No other orientations of the infinite layer phase were observed by four-circle XRD. ${ }^{9,12}$ Typical values for the $c$-axis ranged between 3.43 and $3.47 \AA$. The $a$-axes generally had the same dimensions as the $\mathrm{SrTiO}_{3}$ template, i.e. $a \simeq 3.91 \AA$, relatively independent of the IL composition. We assume that this correspondence results from the prominent role of epitaxy in stabilizing the IL phase. In a preliminary study, however, we have determined that epitaxial films of the IL compound also may be grown on $(100) \mathrm{KTaO}_{3}$, which has a lattice parameter of $3.99 \AA$. The in-plane lattice parameter for a $\mathrm{SrCuO}_{2}$ film on this substrate material was $a=3.95 \AA$. This observation indicates that there may exist a range of lattice parameters suitable for epitaxial stabilization of the IL phase.

Resistivity measurements were performed with a standard four-probe technique, using spring loaded contact pins pressed onto sputter deposited gold contact pads. Hall and thermopower measurements were performed to determine the sign of the majority charge carriers. ${ }^{18,19}$ Information about the nature of the carriers also was derived from examination of the variation in room temperature resistivity induced by oxidation-reduction anneals at moderately low temperatures $\left(300-350^{\circ} \mathrm{C}\right)$. The anneals ${ }^{12}$ were performed in flowing mixtures of oxygen and argon at a total pressure of $1.0 \mathrm{~atm}$. Because oxygen traps electrons, hole-like and electron-like contributions to the total conductivity may lead to distinct dependencies of the resistivity on the oxygen content, reversibly controlled via the low temperature anneals. A close correspondence between results from the oxygen exchange experiments and the more direct Hall and thermopower measurements was observed throughout this investigation, with the additional observation that for some films, the majority charge carriers changed sign as a function of the oxygen content (electron-doped after reduction, hole-doped after oxidation). In plots of the resistivity as a function of the oxygen partial pressure such sign reversal may manifest itself as a nonmonotonic variation, reaching a maximum at some intermediate oxygen pressure. 


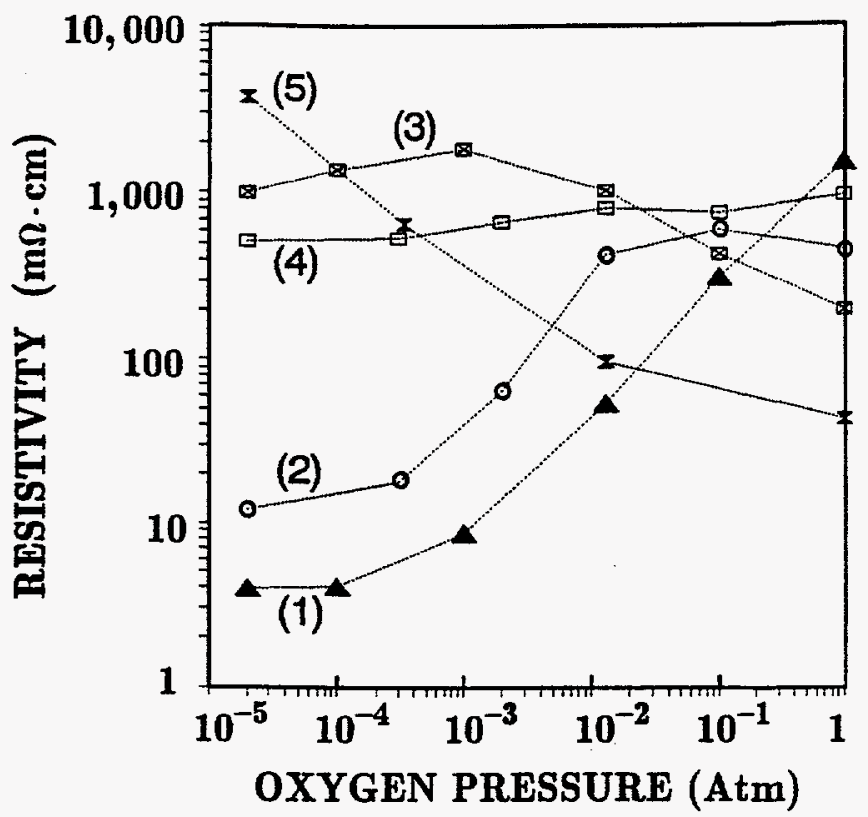

Fig. 1 Variation of the resistivity at $300 \mathrm{~K}$ with the oxygen partial pressure during low temperature oxidation-reduction annealing $\left(350^{\circ} \mathrm{C}\right)$ for codeposited $\mathrm{Sr}_{\mathrm{z}} \mathrm{CuO}_{2} \pm \delta$ infinite layer films: (1) $\mathrm{z}=0.85,(2) \mathrm{z}=1.0,(3) \mathrm{z}=1.2 ; \quad(1)-(3)$ : $550^{\circ} \mathrm{C} / 2 \mathrm{mTorr} ;(4) \mathrm{z}=1.0\left(550^{\circ} \mathrm{C} / 200 \mathrm{mTorr}\right)$; (5) $\mathrm{z}=1.0\left(500^{\circ} \mathrm{C} / 200 \mathrm{mTorr}\right)$.

\section{CARRIER DOPING IN CODEPOSITED INFINITE LAYER FILMS}

The effects of $\mathrm{Sr} / \mathrm{Cu}$ composition and growth conditions on the carrier doping in codeposited films grown by single target laser ablation ${ }^{20}$ are summarized in Fig. 1. Here we have plotted the room temperature resistivity as a function of the oxygen partial pressure during oxidation-reduction annealing at $300-350^{\circ} \mathrm{C}$. It is seen that the resistivity changes for the various films are not the same and may exhibit slopes of opposite sign, evidence for the existence of different types of dopants (defects) in the films. Especially low resistivities were observed for Sr deficient compositions of the IL compound, here represented for a film with $z=0.85$ (curve 1), and for films grown at low oxygen pressures (curve 2), both "as grown" and after post-growth reduction. The resistivity increase after post oxidation indicates that these films are electron-doped. Higher resistivities and hole-doped behavior are observed for films grown at lower temperatures (curve 5) and films with a $\mathrm{Sr}$ rich composition (curve 3).

The observation of electron doping upon $\mathrm{Sr}$ vacancy incorporation is unexpected and contradicts previous reports for high-pressure synthesized bulk ceramics. ${ }^{21,22} \mathrm{Sr}$ vacancies were expected to provide holes, although the possibility of hole-doping in the absence of apical oxygen (or halogens) previously has not been demonstrated. The most likely origin of this electron-doped behavior are oxygen vacancies in the copper-oxygen sheets, which should thus be written as $\mathrm{CuO}_{2-\delta}$. It is plausible that the oxygen vacancies are incorporated to compensate for the $\mathrm{Sr}$ vacancies, rather than lead to a higher valence for $\mathrm{Cu}$. Consistent with this idea, the magnitude of the oxygen induced resistivity variation is largest for the $\mathrm{Sr}$ deficient film of curve 1, suggesting the existence of vacant oxygen sites.

\section{LAYER-BY-LAYER GROWTH OF $\mathrm{SrCuO}_{2}$ FILMS}

A highly controlled method for synthesizing the IL compound is through the layer-by-layer growth (ALE) of sequentially deposited $\mathrm{Sr}(\mathrm{O})$ and $\mathrm{CuO}$ molecular monolayers. During co-deposition, the $\mathrm{Sr}$ and $\mathrm{Cu}$ atoms arrive simultaneously on the substrate surface and the lattice structure is formed by the spontaneous ordering of both constituents (and oxygen). Defects introduced during this ordering process may be suppressed during ALE. On the other hand, the growth of artificially imposed stacking sequences may be in competition with the spontaneous ordering tendencies if the thermodynamic growth parameters are essentially the same (as is the case in the present study). Thus, by monitoring the growth via RHEED and introducing deviations in the deposition sequence, unique insights may be obtained into the natural tendencies for defect formation and carrier doping. 


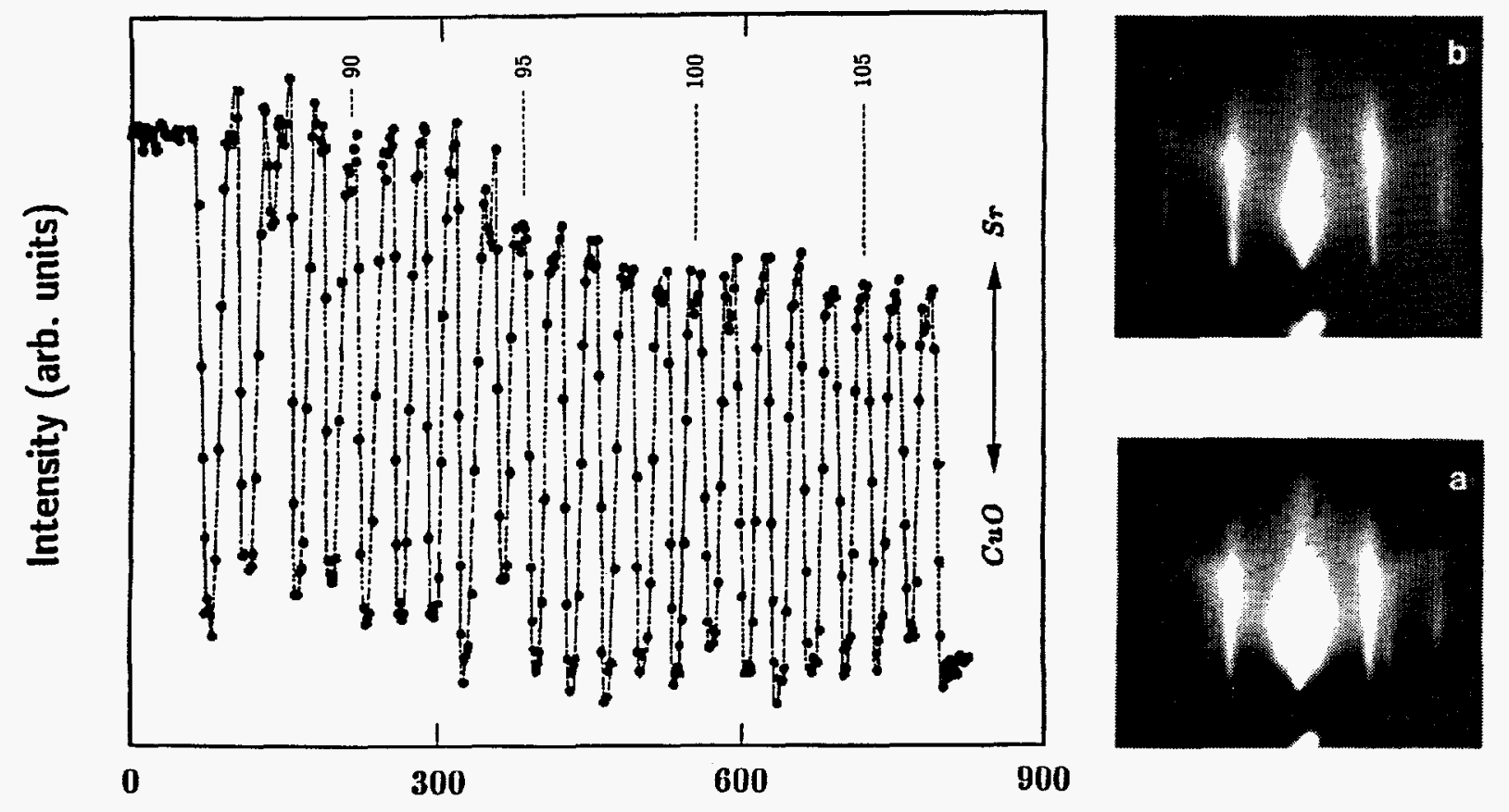

Time (s)

Fig. 2 Intensity variations of the specular reflection in RHEED induced by the alternate deposition of $\operatorname{Sr}(\mathrm{O})$ and $\mathrm{CuO}$ monolayers during layer-by-layer growth of $\mathrm{a} \mathrm{Sr}_{\mathrm{z}} \mathrm{CuO}_{2}$ infinite layer film. The intensity increases during $\operatorname{Sr}(\mathrm{O})$ deposition and decreases during $\mathrm{CuO}$ deposition. Numbers indicate the approximate number of deposited $\mathrm{Sr}_{\mathrm{z}} \mathrm{CuO}_{2}$ unit cell layers. RHEED patterns correspond to the minima ("a": after CuO deposition) and maxima (" $\mathrm{b}$ ": after $\mathrm{Sr}(\mathrm{O})$ deposition) of the oscillations, respectively. The electron beam is incident along the $[100]$ azimuth of the substrate at a glancing angle of approximately $1-2^{\circ}$.

Fig. 2 illustrates intensity variations of the specularly reflected electron beam along the central $(00)$ rod in the RHEED pattern observed during the ALE growth of a $\mathrm{Sr}_{\mathbf{z}} \mathrm{CuO}_{2}$ film. The intensity oscillations are induced by the periodic appearance of a $\mathrm{CuO}_{y}$ (minimum of the oscillation) and $\operatorname{Sr}(\mathrm{O})$ surface layer (maximum of the oscillation), initiated by operator-controlled interruptions of the deposition process and target exchange. Typical RHEED patterns at the bottom ("a") and top ("b") of the oscillations are also illustrated in Fig. 2. These patterns and especially the appearance of extra, incommensurate streaks after $\mathrm{CuO}$ deposition were used to guide the layer-bylayer growth process. ${ }^{23}$ The amount of material deposited per period of the deposition cycle corresponds to approximately one unit cell layer of $\mathrm{Sr}_{\mathrm{z}} \mathrm{CuO}_{2}$. Although RHEED oscillations with a similar period also may be observed in a codeposition process, it is important to note that the origin of the oscillations is different. During codeposition, the RHEED oscillations are induced by periodic variations in the density of unit cell high steps on the film surface. ${ }^{17}$ The oscillations during ALE are induced by the appearance of different chemical species at the topmost surface, induced by the sequential monolayer depositions. ${ }^{24}$ Intensity oscillations also occur when consistently less than a full monolayer of both $\mathrm{CuO}$ and $\operatorname{Sr}(\mathrm{O})$ are deposited, in which case the accumulated thickness per oscillation is less than one unit cell. ${ }^{12}$ Calibration of the deposition process (the number of laser pulses per $\mathrm{CuO}$ and $\operatorname{Sr}(\mathrm{O})$ monolayer) was achieved via an iterative process whereby the thickness of several films $(\sim 70 \AA)$ was evaluated from the finite-size diffraction peaks in a normal $\theta-2 \theta$ XRD pattern. In optimized cases, the correspondence between the number of RHEED oscillations and the film thickness (expressed in multiples of $c$ ) was within a few percent.

A remarkable observation, described in more detail in ref. 12 , is that films grown with a strictly periodic CuO$\mathrm{Sr}(\mathrm{O})$ layering sequence generally behaved as electron-doped semiconductors. Moreover, inspection of the relative intensities of the (001) and (002) IL phase XRD peaks indicated that the films had a Sr deficient ${ }^{20}$ matrix (up to $20 \% \mathrm{Sr}$ deficient). Since no independent measure of the surface composition or absolute calibration of deposition 
rates was used in these ALE experiments, it is possible that deviations from the perfect $\mathrm{Sr}: \mathrm{Cu}=1: 1$ stoichiometry resulted from the operator imposed constraint that the RHEED oscillations be weakly damped and the RHEED pattern remain streaky, indicative of a stepped surface. Empirically it was observed that consistently maintained deviations from the compositional balance established during this layer-by-layer growth, either for $\operatorname{Sr}(\mathrm{O})$ or $\mathrm{CuO}$, rapidly lead to the appearance of spots in the RHEED pattern, indicating secondary phase formation on the film surface. The tendency towards $\mathrm{Sr}$ deficiency thus may be a natural tendency for the $\mathrm{SrCuO}_{2}$ IL lattice under the employed growth conditions. This example illustrates how monitoring of the growth mechanism during ALE may contribute to an understanding of the tendencies towards defect formation and carrier doping.

The combined observations of a $\mathrm{Sr}$ deficient matrix and electron-doped transport properties for the periodic ALE films are consistent with those for the codeposited films ablated from a $\mathrm{Sr}$ deficient $\mathrm{Sr}_{\mathbf{z}} \mathrm{CuO}_{2}$ target. Likewise, it was observed that deliberate additions of extra $\operatorname{Sr}(\mathrm{O})$ monolayers during the ALE process induced hole-doped transport properties of the IL films (layering sequence .. Sr-CuO-Sr-Sr-CuO- . .). Attempts to insert extra SrO monolayers between blocks of codeposited $\mathrm{SrCuO}_{2}$ to form compounds in the homologous series $\mathrm{Sr}_{n+1} \mathrm{Cu}_{n} \mathrm{O}_{2 n+1}$ will be described in more detail in section 7. The ALE experiments strongly suggest that SrO defect layers can act as charge reservoir layers in the IL compound. Indeed, arrays of SrO double layers, periodically and aperiodically stacked along the $c$ axis, have been identified by TEM in high-pressure synthesized offstoichiometric compositions ${ }^{22}$ of the IL compound $(\mathrm{Sr}, \mathrm{Ca}) \mathrm{CuO}_{2}$. Because of these intergrowth defects, it has remained obscure to date whether hole-doping and superconductivity may be induced purely by $A$-ion vacancies. ${ }^{21}$

\section{ELECTRON MICROSCOPY}

Cross sectional images of IL films grown with the ALE technique are presented in Fig. 3 . The images were obtained with a VG Microscopes HB501UX Z-contrast STEM, operated at an acceleration voltage of $100 \mathrm{kV}$ with a probe size of $2.2 \AA$. A special feature of this microscope is the capability to resolve compositional differences between neighboring atom columns through contrast differences in the dark field image. ${ }^{25}$ In the case of the $\mathrm{SrCuO}_{2} \mathrm{IL}$ compound, this means that $\mathrm{Sr}$ and $\mathrm{Cu}$ columns along the $c$-axis (plan-view) or along the $a$-axis (cross sectional view) can be uniquely identified: a bright feature indicates $\mathrm{Sr}$ while a less-bright feature indicates $\mathrm{Cu}$. The positions of $\mathrm{Sr}$ and $\mathrm{Cu}$ atoms have been marked in the image of Fig. 3a, which was obtained for a film grown with periodic depositions of $\mathrm{Sr}(\mathrm{O})$ and $\mathrm{CuO}$ monolayers. Electron transparent specimens were prepared by mechanical polishing to $25 \mu \mathrm{m}$, followed by argon ion milling at $2-5 \mathrm{keV}$ on a liquid nitrogen cooled stage. The tetragonal IL structure with $a \simeq 3.9 \AA$ and $c \simeq 3.45 \AA$ can clearly be identified from the location of the $\mathrm{Sr}$ dots (columns) in Fig. 3a. Extended defects cannot be observed in this image.
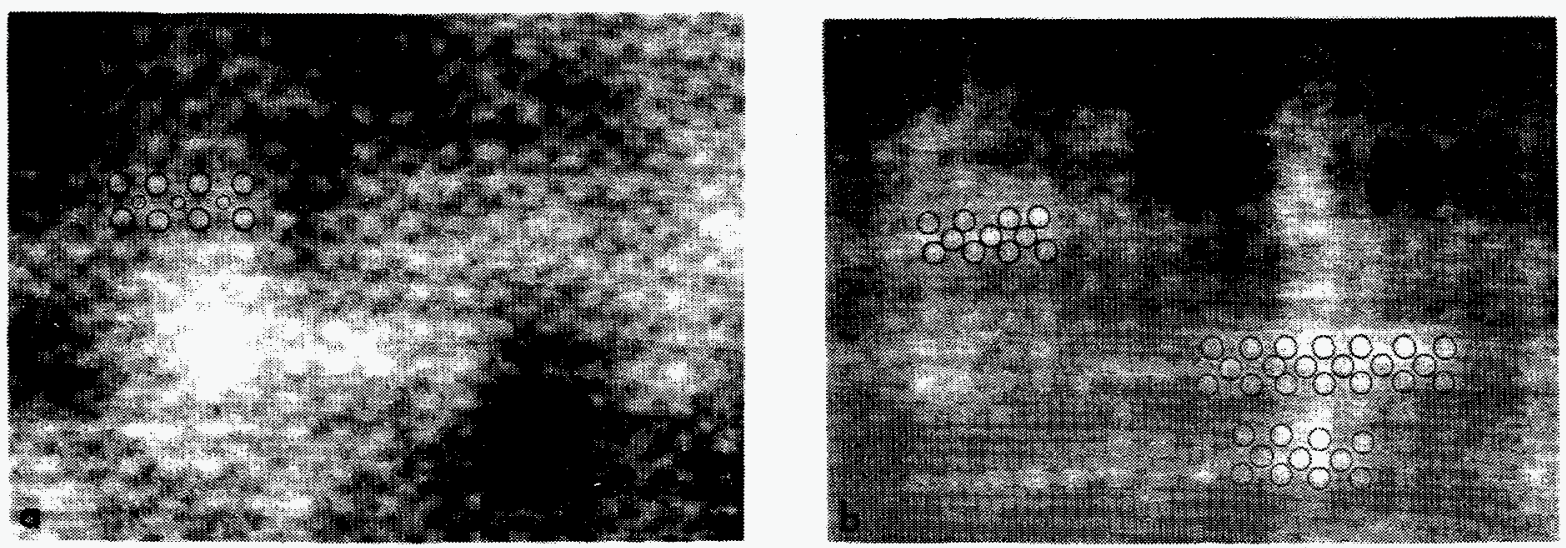

Fig. 3 Cross section Z-contrast STEM images of $\mathrm{Sr}_{\mathrm{z}} \mathrm{CuO}_{2}$ infinite layer films grown by ALE: (a) with periodic depositions of $\mathrm{Sr}(\mathrm{O})$ and $\mathrm{CuO}$ monolayers, and (b) with inserted SrO defect layers. In (a) Sr and $\mathrm{Cu}$ positions have been marked by large and small circles, respectively. Sr positions in extended defects have been marked in (b). Note the formation of triple layers and small lateral dimensions of the SrO defects. 

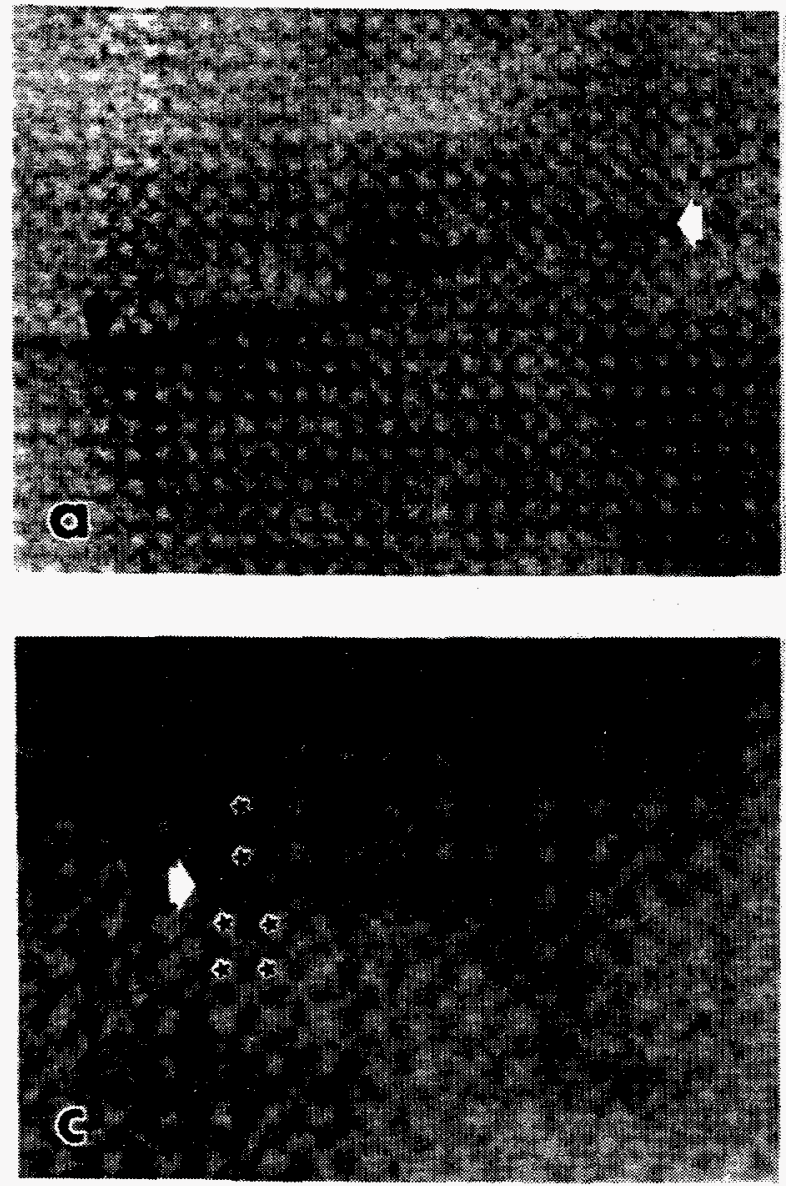

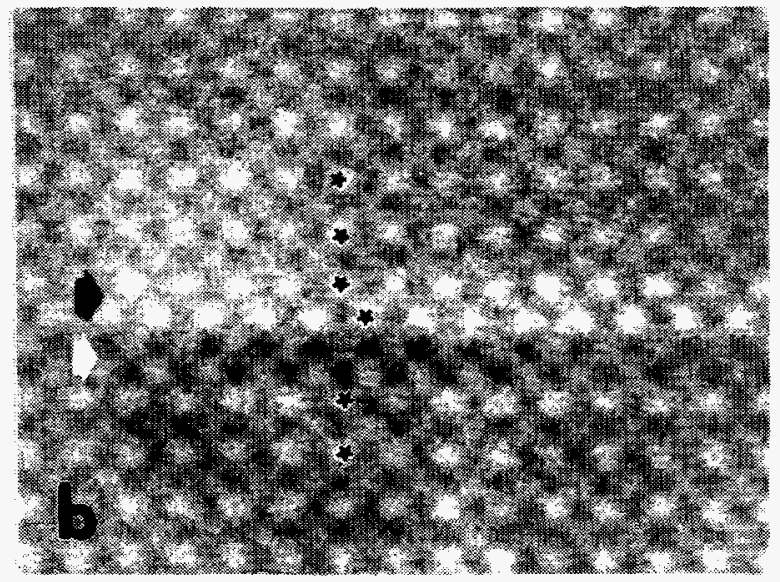

Fig. 4 Plan-view Z-contrast STEM images of a codeposited $\mathrm{Sr}_{0.8} \mathrm{CuO}_{2}$ infinite layer film: (a) general view of an extended defect composed of $\mathrm{SrO}$ (bright) and $\mathrm{CuO}$ (dark) double layers, (b) enlargement of the left side of the defect where $\mathrm{SrO}$ and $\mathrm{CuO}$ double layers meet, (c) enlargement of the $\mathrm{CuO}$ defect at the center right. Dark and light arrows indicate the $\mathrm{SrO}$ and $\mathrm{CuO}$ defects, respectively. $\mathrm{Sr}$ positions across the defect planes have been marked with asterisks in (b) and (c).

By contrast, the image of Fig $3 \mathrm{~b}$ was obtained for an ALE film containing deliberately inserted submonolayers of SrO. Sr rich defects are readily observed in this image, marked by a cubic arrangement of the Sr columns. Rather than forming flat defect layers with a thickness of two SrO monolayers, the extra Sr has been incorporated in the form of islands with a thickness of three monolayers. As we will discuss below, this island-like intergrowth is consistent with the island-like growth mechanism observed in situ by RHEED. The correlation with the transport properties suggests that the hole-doping originates from apical oxygen atoms located in these SrO defects. Tripled $\mathrm{Sr}_{3} \mathrm{O}_{2+\mathrm{x}}$ defect layers recently also have been identified by Zhang et al. ${ }^{26}$ in superconducting, high-pressure synthesized bulk specimens. As argued by the authors, the presence of an extra SrO plane in the defect layer may enhance the number of apical oxygen atoms compared to $\mathrm{Sr}_{2} \mathrm{O}_{1+\mathrm{x}}$ double layers. A recent neutron diffraction study ${ }^{27}$ of the superconducting compound $\mathrm{Sr}_{2} \mathrm{CuO}_{3+\epsilon}$, however, indicates the existence of a substantial oxygen deficiency on the $\mathrm{CuO}_{2-\delta}$ sheets $(\delta \simeq 1)$ with fully oxidized $\mathrm{Sr}_{2} \mathrm{O}_{1+x}$ blocking layers $(\mathrm{x} \simeq 1)$. Charge neutrality constraints mandate that the oxygen sites in the copper-oxygen planes and SrO double layers cannot be fully occupied simultaneously. In the case of the films with extra SrO defects, no superconductivity was observed in the "as grown" state nor after low temperature oxidation; however, a gradual semiconductor-metal transition could be induced by high-temperature annealing (section 7 ).

Plan-view images of a codeposited $\mathrm{Sr}_{0.8} \mathrm{CuO}_{2}$ film are shown in Fig. 4. Previous studies ${ }^{28,29}$ have shown that IL films may exhibit high densities of planar defects perpendicular to the substrate surface. The defects have been identified as $c / 2$ stacking faults in the IL lattice (with an additional translation of $a \sqrt{2} / 2$ along [110]), resulting in "walls" of rocksalt SrO double layers parallel to the c-axis. As illustrated in Fig. 4, two additional perpendicular defects have been identified with the Z-contrast STEM technique. In Fig. 4a, a meandering defect consisting of a bright and a dark band is observed in the left side of the image. The higher magnification image of Fig. $4 \mathrm{~b}$ indicates that this defect consists of two $\mathrm{Sr}$ columns (bright dots) and two $\mathrm{Cu}$ columns (gray dots), comprising one unit cell 

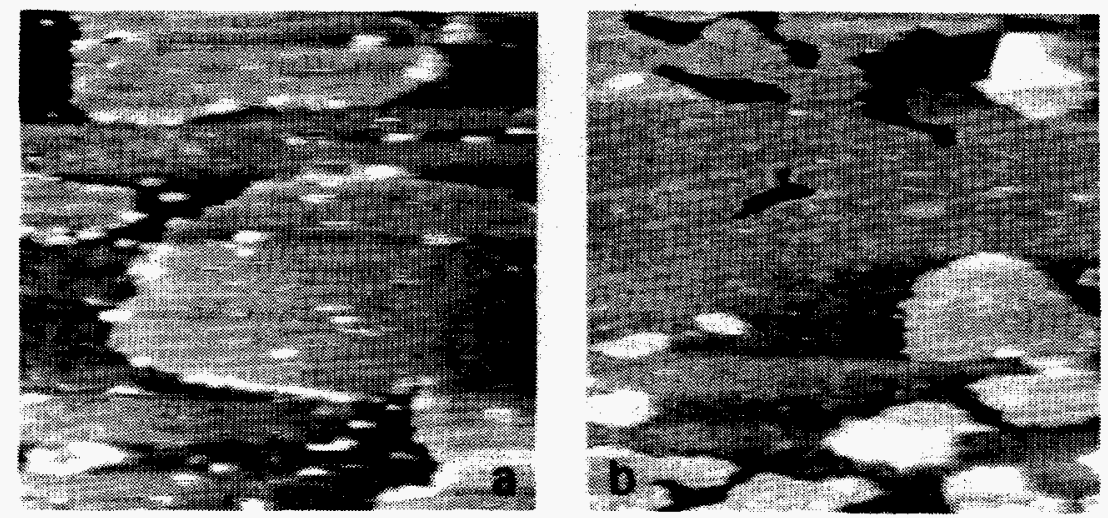

Fig. 5 Constant current STM images of infinite layer films grown by co-deposition in the laser-MBE system: (a) $\mathrm{SrCuO}_{2}$ and (b) $\mathrm{Sr}_{0.95} \mathrm{La}_{0.05} \mathrm{CuO}_{2}$. The images were obtained with a sample bias of $2 \mathrm{~V}$ (empty states) and a tunneling current of $10 \mathrm{pA}$. The image areas are $880 \AA$ (horizontal) by $1690 \AA$. The difference in vertical height between terraces of different shadings is one unit cell $(\sim 3.4 \AA)$.

(along $b$ ) of the stable orthorhombic $\mathrm{SrCuO}_{2}$ phase. ${ }^{30}$ Near the center of the image, this defect splits in two separate branches of bright and dark contrast which seem to propagate independently. The brighter defect (towards the top of Fig. 4a) consists of two $\mathrm{SrO}$ columns and probably is identical to the previously reported $c / 2$ stacking faults. The darker defect (Fig. 4c), on the other hand, indicates the intergrowth of a double CuO chain and has not been reported previously for IL films. This chain-like configuration is similar to that reported by Hiroi et al. ${ }^{31}$ for the $\mathrm{Sr}_{n-1} \mathrm{Cu}_{n+1} \mathrm{O}_{2 n}$ homologous series of semiconductors, observed as a byproduct of the IL phase in high-pressure synthesized ceramics under reducing conditions. It is tempting to attribute the large $\mathrm{Sr}$ deficiencies $(\mathrm{Sr} / \mathrm{Cu} \geq 0.7)$ that can be accommodated in IL films ${ }^{20}$ to the intergrowth of these defects. On the other hand, it is remarkable that Sr-rich extended defects are formed even at these Sr-deficient compositions. While no significant effect on the carrier doping is expected from either of the perpendicular defects, their potential role as "sinks" for compositional deviations in the IL matrix has to be taken into account when correlating the carrier doping to the $\mathrm{Sr} / \mathrm{Cu}$ composition.

\section{SCANNING TUNNELING MICROSCOPY ON QUENCHED GROWTH SURFACES}

The surface morphology of IL films grown with the laser-MBE technique was studied with a UHV scanning tunneling microscope. ${ }^{32}$ The STM chamber is connected to the growth chamber via a gate valve, making it possible to study quenched growth surfaces without exposure to the ambient air. ${ }^{33}$ The films were deposited from composite targets on semiconducting $0.01 \% \mathrm{Nb}$-doped $\mathrm{SrTiO}_{3}$ substrates in a low pressure of $\mathrm{NO}_{2}$, similar to the previous laserMBE experiments. RHEED was used to monitor the growth process and terminate the deposition at a predetermined moment. Typical film thicknesses ranged between $15-20 \AA$.

STM images for a stoichiometric $\mathrm{SrCuO}_{2}$ film and $5 \%$ La-doped film $\left(\mathrm{Sr}_{0.95} \mathrm{La}_{0.05} \mathrm{CuO}_{2}\right)$ are shown in Fig. 5a and $5 \mathrm{~b}$, respectively. The images were obtained at room temperature using tungsten or $\mathrm{Pt} / \mathrm{Ir}$ tips at a sample-tip bias voltage of $2 \mathrm{~V}$ (empty states). Atomically flat terraces are observed with an island-like morphology, representing consecutive unit-cell layers of the IL compound. Height scans confirm that the steps are approximately $3.4 \AA$ high, corresponding to the IL $c$-axis length. Typically, only two or three terrace levels were observed at this magnification, indicating a layer-by-layer growth mechanism.

While the surface of the La-doped film contains few irregularities, the surface of the stoichiometric film appears to contain large numbers of "particles," located mostly near the step edges. We assume that these particles represent $\mathrm{Sr}$ atoms (or small clusters of $\mathrm{Sr}$ atoms) which have been expelled from the IL matrix. In a parallel study of the $\mathrm{Sr}_{1-\mathrm{x}} \mathrm{Ca}_{\mathbf{x}} \mathrm{CuO}_{2}$ system, Koguchi et al. ${ }^{34}$ recently reported a clear trend in the density of these particles with the $\mathrm{Sr} / \mathrm{Ca}$ ratio: Practically no particles were present on the surface of a film containing $30 \% \mathrm{Sr}$ and $70 \% \mathrm{Ca}(\mathrm{x}=0.7)$, however, the particle density increased steadily with the $\mathrm{Sr}$ concentration. In the $\mathrm{Sr}_{1-\mathrm{x}} \mathrm{Ca}_{\mathrm{x}} \mathrm{CuO}_{2}$ solid solution 
system, a narrow range of stable compositions ${ }^{14}$ exists for $\mathrm{x} \simeq 0.86$. With increasing Sr concentration, an interfacial stress builds up between the $\mathrm{CuO}_{2}$ sheets and the intermediate $\mathrm{Sr}_{1-\mathrm{x}} \mathrm{Ca}_{\mathbf{x}}$ ion layers because of the larger average $A$ ion size. ${ }^{2}$ This stress, which is compressive for the $\mathrm{Sr}_{1-\mathrm{x}} \mathrm{Ca}_{\mathrm{x}}$ layer and tensile for the $\mathrm{CuO}_{2}$ sheets, is the intrinsic cause of the metastable nature of the $\mathrm{Sr}(\mathrm{Ba})$ rich compositions of the IL compound. It is plausible that this stress is relieved through the incorporation of $\mathrm{Sr}$ vacancies under mildly oxidizing conditions. Thus, a somewhat $\mathrm{Sr}$ and oxygen deficient matrix remains, with the Sr excess initially expelled to the surface and eventually in extended defects. The nearly complete absence of particles on the La-doped film surface (Fig. 5b) supports this model: The additional positive charge of the substituted $\mathrm{La}^{3+}$ ions diminishes the tendency for oxygen vacancy formation and thus the formation of $\mathrm{Sr}$ vacancies. Moreover, the $\mathrm{Cu}-\mathrm{O}$ bond is slightly stretched due to electron-doping from the trivalent La atoms. ${ }^{3,15}$ Together with the slightly smaller $\mathrm{La}^{3+}$ radius, this would diminish the interfacial stress.

In selected cases, STM images at atomic resolution were obtained for the IL films. ${ }^{34}$ The images contain atomic square lattices of bright dots with randomly distributed point defects. The period of the dots is $4 \AA$, corresponding to the $a$-axis of the IL lattice. Moreover, the images exhibit an interesting dependence on the bias voltage, reflecting spatial variations in the electronic structure of the IL compound. In related spectroscopy experiments (STS), a clear gap in the tunneling current is observed around zero bias voltage. ${ }^{35}$ The gap width and the position of the Fermi energy inside the gap depends on the IL composition and the final oxidation/reduction treatment. Since the states contributing to the tunneling current are predominantly located in the copper-oxygen sheets, it follows that the carrier doping may be studied at the atomic level with the STM/STS technique, unperturbed by nonsuperconducting charge reservoir layers. We will report on the doping dependent STS experiments in a forthcoming publication.

\section{INFINITE LAYER FILMS WITH PERIODICALLY INSERTED DEFECT LAYERS}

The conspicuous absence of hole-doping in the $\mathrm{Sr}$-deficient films indicates that charge reservoir layers containing apical oxygen (halogen) atoms are needed to induce (hole-doped) superconductivity. In recent publications ${ }^{36,37}$ on
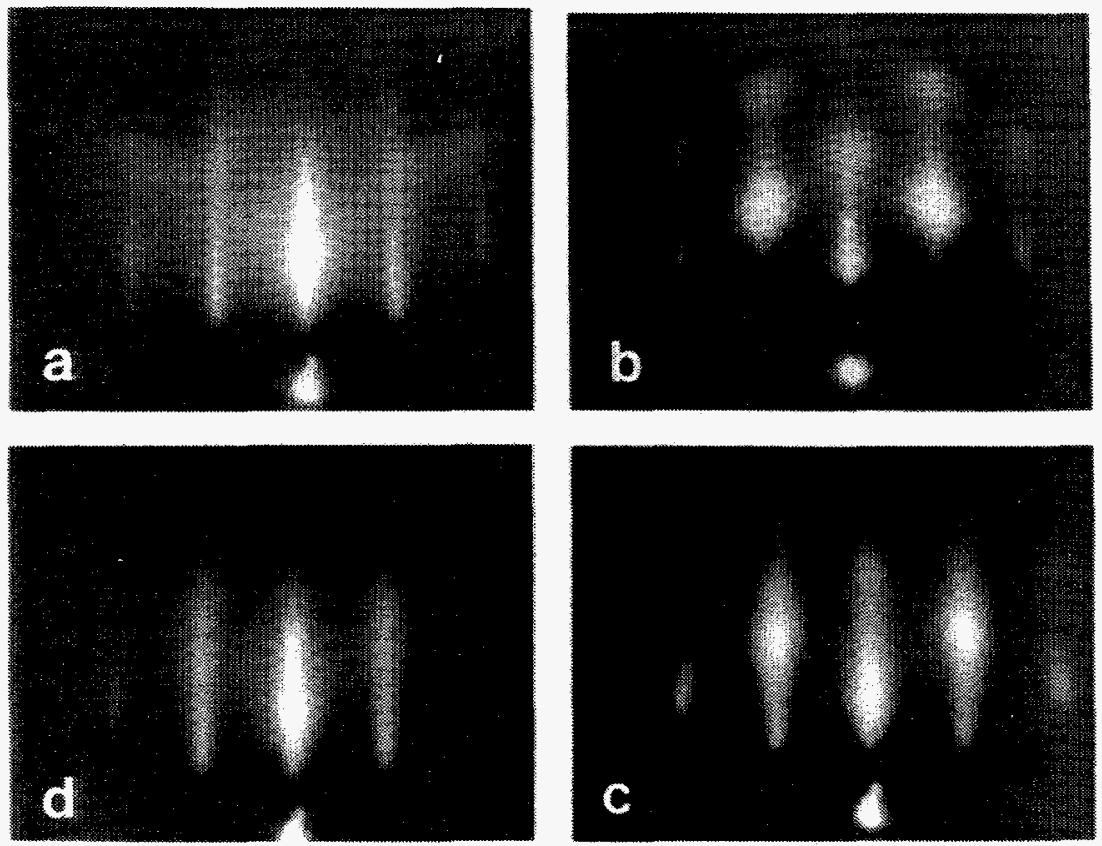

Fig. 6 Sequence of RHEED patterns depicting the island-like intergrowth of a SrO defect layer in the infinite layer compound under moderately oxidizing conditions: (a) film surface after co-deposition of 10 $\mathrm{SrCuO}_{2}$ unit cell layers on $\mathrm{SrO}$ terminated $\mathrm{SrTiO}_{3}$, (b) after deposition of $1 \mathrm{SrO}$ monolayer, (c) after subsequent deposition of $1 \mathrm{SrCuO}_{2}$ unit cell layer, (d) after deposition of $7 \mathrm{SrCuO}_{2}$ unit cell layers (total) on the $\mathrm{SrO}$ defect layer. The incident electron beam is parallel to the in-plane [100] direction. 
high-pressure synthesized bulk ceramics, superconductivity with $T_{c}$ up to $100 \mathrm{~K}$ has been reported for the "Ruddlesden-Popper" phases $\mathrm{Sr}_{n+1} \mathrm{Cu}_{n} \mathrm{O}_{2 n+1}$, consisting of arrays of the IL phase with periodically intergrown $\mathrm{SrO}$ extra lattice planes. Considering the "incomplete" nature of the oxygen sublattice, ${ }^{27}$ the origin of the superconductivity in these compounds is presently unclear. It has been suggested, however, that the superconductivity in offstoichiometric bulk specimens of the IL compound may be due to the intergrowth of these phases. ${ }^{22}$

We have used the laser-MBE technique to form thin films of the $\mathrm{Sr}_{n+1} \mathrm{Cu}_{n} \mathrm{O}_{2 n+1}$ phases $(\mathrm{n}=3-9)$ via deposition controlled insertion of the extra $\mathrm{SrO}$ lattice planes. ${ }^{13}$ The $\left[\mathrm{SrCuO}_{2}\right]_{n}$ IL blocks were formed by codeposition from a composite target. The extra SrO monolayers were ablated from a metallic $\mathrm{Sr}$ disk. The deposition conditions $\left(\mathrm{NO}_{2}\right.$ pressure and substrate temperature) were kept the same as for the $\mathrm{SrCuO}_{2}$ IL films. A sequence of RHEED patterns depicting the intergrowth of a SrO "layer" is presented in Fig. 6. Pattern 6a was obtained after the deposition of 10 unit cell layers of the IL compound. The pattern is streaky, indicating a smooth surface. Distinct spots appeared in the RHEED pattern upon deposition of the extra SrO monolayer (Fig. 6b). The spots are similar to those observed during ALE film growth upon continued $\mathrm{Sr}(\mathrm{O})$ deposition and they indicate that the $\mathrm{SrO}$ monolayers do not optimally wet the underlying structure, but rather form islands. The spots disappear only partially after overgrowth with the next $\left[\mathrm{SrCuO}_{2}\right]_{\mathbf{n}}$ block (Fig. 6c, d). Repetition of this cycle to form the $\mathrm{SrO}-\left[\mathrm{SrCuO}_{2}\right]_{\mathbf{n}}$ superlattice structure rapidly leads to a permanently spotty RHEED pattern, indicating a disordered surface structure. The growth can be improved by reducing the $\mathrm{NO}_{2}$ supply; however, as described in section 3 , a lower oxidation pressure tends to produce electron-doped transport properties. We attribute the island-like growth properties to the charge neutrality conflict that would arise upon full oxidation of the $\mathrm{SrO}$ double layers and the underlying copper-oxygen sheet: By forming islands the contact area is reduced and thus the cause of the charge neutrality conflict.

Despite the imperfect growth of the SrO layers, the $\mathrm{Sr}_{n+1} \mathrm{Cu}_{n} \mathrm{O}_{2 n+1}$ films typically were hole-doped (as grown). A semiconductor-metal transition, in some cases accompanied by the onset of superconductivity, could be induced by high temperature annealing (Fig. 7). A key parameter for inducing the metallic behavior proved to be cooling rate at the end of the anneals. Conductivity enhancements only occurred when the samples were cooled rapidly (performed by sliding the quartz sample holder out of the furnace hot zone), whereas slower cooling procedures, or reanneals at lower temperatures, led to drastic resistance increases. ${ }^{13}$ Moreover, the conductivity enhancements only occurred for films containing the extra SrO deposits. This is illustrated in Fig. 8 where we have plotted the resistance ratio $\alpha=\mathrm{R}(100 \mathrm{~K}) / \mathrm{R}(300 \mathrm{~K})$ as a function of the annealing temperature for a variety of films. It is seen that a gradual transition from semiconductive $(\alpha>1)$ to metallic $(\alpha<1)$ behavior occurs for the $\operatorname{Sr}_{n+1} \mathrm{Cu}_{n} \mathrm{O}_{2 n+1}$ films containing extra $\mathrm{SrO}$ deposits at an annealing temperature of $\sim 700^{\circ} \mathrm{C}$ (Fig. $8 \mathrm{~b}$ ), but that essentially uncorrelated changes in the shape of the resistivity curves occur for codeposited, electron- or hole-doped films (without the extra SrO deposits, Fig. 8a). The data indicate that the semiconductor-metal transition is uniquely related to the extra $\mathrm{SrO}$ monolayers. In this context it is noteworthy that the IL lattice becomes unstable at high temperature. Partial decomposition into the stable insulating phases $\mathrm{Sr}_{2} \mathrm{CuO}_{3}, \mathrm{Sr}_{14} \mathrm{Cu}_{24} \mathrm{O}_{41}$, and $\mathrm{CuO}$ was observed for several films after annealing at temperatures greater than $700^{\circ} \mathrm{C}$. The exact cause of the transition towards

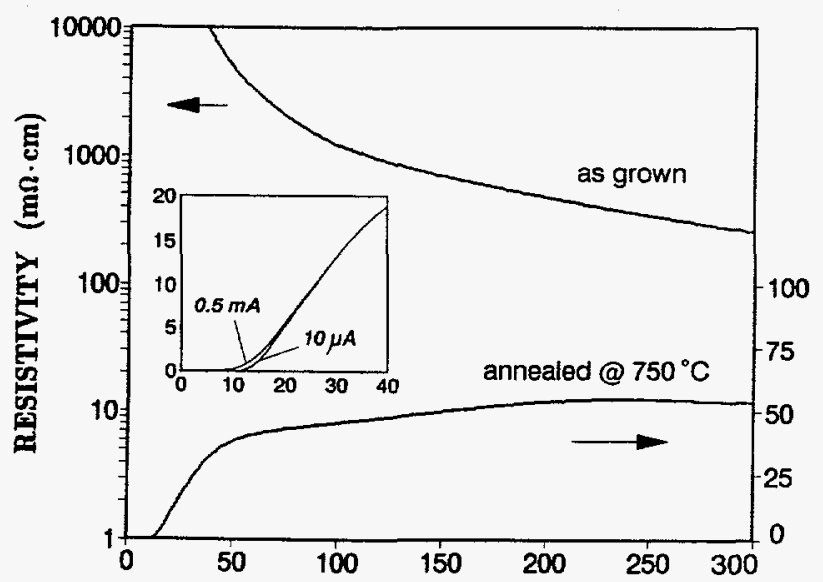

TEMPERATURE (K)
Fig. 7 Temperature dependence of the resistivity for a $\mathrm{Sr}_{n+1} \mathrm{Cu}_{\mathrm{n}} \mathrm{O}_{2 \mathrm{n}+1}$ film with $n=5$, showing semiconductor-to-metal transition and onset of superconductivity after annealing in 1.0 atm oxygen at $750^{\circ} \mathrm{C}$. The inset shows the superconducting transition on an expanded scale for two values of the measuring current. 

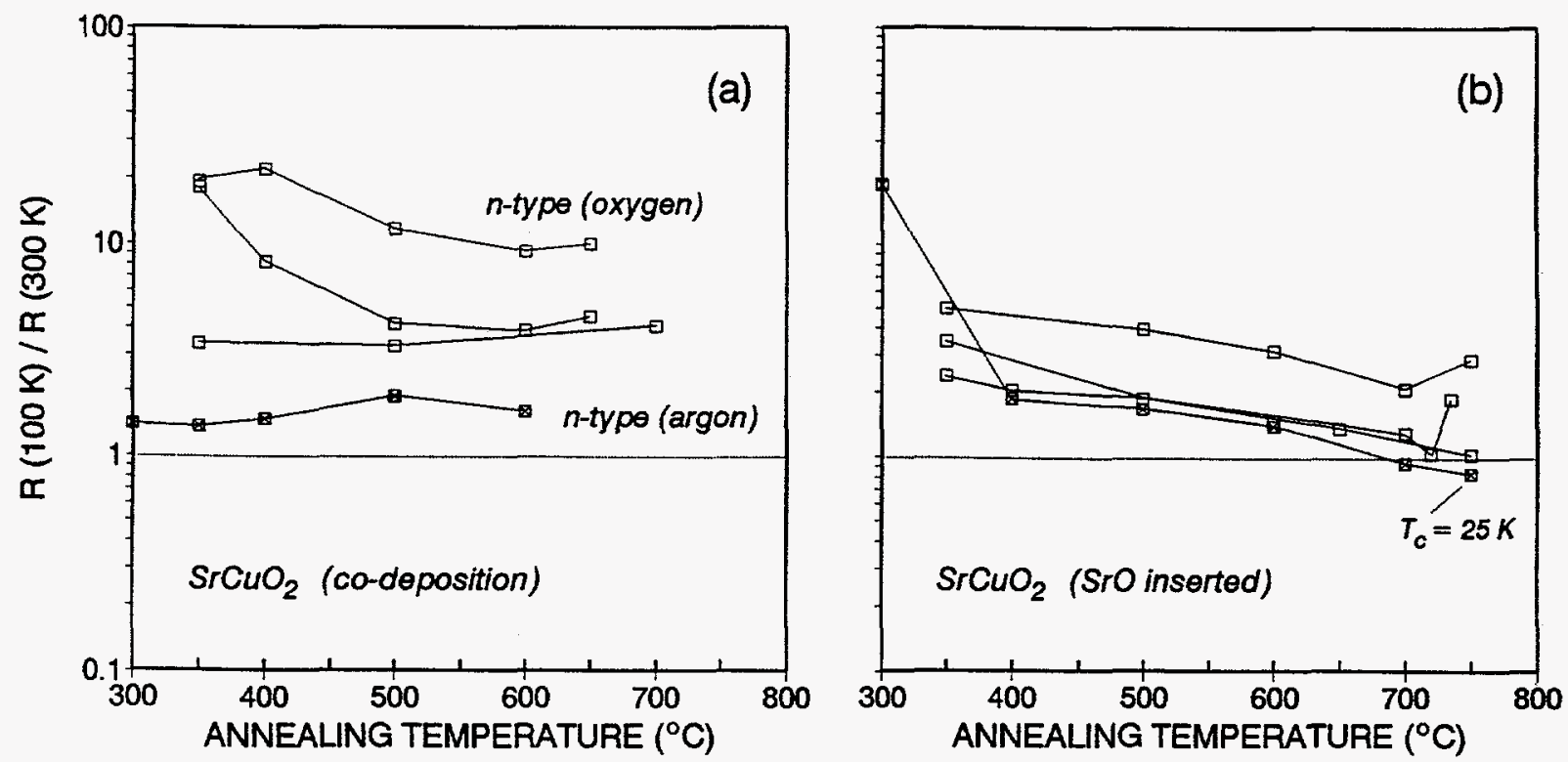

Fig. 8 Variations in the resistance ratio $R(100 \mathrm{~K}) / R(300 \mathrm{~K})$ induced by post-growth anneals at successively higher temperatures for: (a) codeposited $\mathrm{SrCuO}_{2}$ infinite layer films, and (b) $\mathrm{Sr}_{\mathbf{n}+1} \mathrm{Cu}_{\mathbf{n}} \mathrm{O}_{2 n+1}$ films containing artificially inserted $\mathrm{SrO}$ defects. Films labeled n-type were electron-doped; all other films were hole-doped. Except where noted, the anneals were performed in $1.0 \mathrm{~atm}$ oxygen, followed by rapid cooling.

metallic behavior and the onset of superconductivity thus remains obscure. It is plausible, however, that a reordering of oxygen vacancies in the vicinity of the SrO defects, possibly in combination with extra oxygen uptake, ${ }^{38}$ may occur during high-temperature oxidation. The role of the fast cooling rate then is to essentially preserve the high-temperature oxygen sublattice.

\section{CONCLUSIONS}

In this paper we have presented an extensive study of the defect formation and carrier doping in epitaxial films of the IL compound $\mathrm{SrCuO}_{2}$. Information of a complementary nature was obtained from observation of the epitaxial growth mechanism by RHEED and STM, structural characterization by Z-contrast STEM and XRD, electrical transport measurements (resistivity, Hall, and thermopower), and also the response to low temperature oxygen exchange and high temperature annealing. Combined, these data provide a first coherent picture of the growth mechanism of this basic "building block" of the cuprate superconductors. With the synthesis of multilayered derivatives, we anticipate that this information eventually may contribute to a detailed understanding of the defect formation in more complex high- $T_{c}$ compounds, as well.

According to the model derived from these experiments, the most pervasive defects in $\mathrm{SrCuO}_{2}$ films are vacancies on the $\mathrm{Sr}$ and $\mathrm{O}$ sites, leading to electron-doped rather than hole-doped, semiconducting transport properties. The defects, presumably, occur in pairs because of charge neutrality constraints. They are induced by inadequate oxidation and/or the intrinsic lattice mismatch between $\mathrm{CuO}_{2}$ planes and the intermediate $\mathrm{Sr}^{2+}$ layers. The lattice mismatch may be reduced by $\mathrm{Ca}$ substitution, leading to a more insulating parent, or doping with rare earth ions such as $\mathrm{La}$ or $\mathrm{Nd}$, which enhance the electron doping. By extrapolation, $\mathrm{BaCuO}_{2}$ in a strictly IL lattice is unstable and should contain either large numbers of extended defects or a drastically modified oxygen sublattice. We assume that the latter possibility is more realistic and may account for the role of $\left[\mathrm{BaCuO}_{\mathrm{y}}\right]_{\mathrm{m}}(\mathrm{m}=2,3)$ blocks as variable charge reservoir layers in the high- $T_{c}$ cuprates. ${ }^{16,39}$ Hole doping in $\mathrm{SrCuO}_{2}$ films is induced by $\mathrm{Sr}$ atoms expelled from the IL matrix, accommodated in extended defects which may be either parallel or perpendicular to the copperoxygen planes. The distribution between these two orientations probably depends on the growth conditions and the film growth method. For example, in Fig. 1, data were presented for a stoichiometric $\mathrm{SrCuO}_{2}$ film that was grown 
at a relatively low oxygen pressure (curve 2). This film was electron-doped after reduction, indicating formation of a $\mathrm{Sr}$ and $\mathrm{O}$ deficient matrix. We assume that the SrO defects in this case are predominantly oriented perpendicular to the substrate surface and do not contribute significantly to the doping mechanism. Previous electron microscopy studies ${ }^{28,29}$ indicate that the density of such perpendicular defects in codeposited $\mathrm{SrCuO}_{2}$ films may indeed be rather high. On the other hand, larger fractions of parallel extended SrO defects may be induced via the ALE technique, introducing apical oxygen sites. The readily observed hole-doped transport properties for such films agree well with the previously established correlation between carrier doping and the $\mathrm{Cu}-\mathrm{O}$ coordination number.

A central role in this defect model is played by the in situ oxidation during epitaxial growth. In addition to pressure, the oxidation indeed forms an important difference with high-pressure synthesis techniques, which often involve the use of oxidizing agents during the high temperature sintering. For epitaxial films, the maximum oxygen activity is limited by the vacuum ambient of the growth chamber. The issue of in situ oxidation previously has been successfully solved for films of stable high- $T_{c}$ compounds such as $\mathrm{YBa}_{2} \mathrm{Cu}_{3} \mathrm{O}_{6+\mathrm{x}}, \mathrm{La}_{2-\mathrm{x}} \mathrm{Sr}_{\mathrm{x}} \mathrm{CuO}_{4}$, or $\mathrm{Bi}_{2} \mathrm{Sr}_{2} \mathrm{CaCu}_{2} \mathrm{O}_{8}$, which generally exhibit properties similar to those of their bulk counterparts. The necessary growth temperatures $\left(700-800^{\circ} \mathrm{C}\right)$, however, are significantly higher than those accessible for epitaxial films of the IL compound (500$600^{\circ} \mathrm{C}$ ). Because of reduced reaction kinetics, it is possible that the oxidation techniques developed for high temperature in situ film growth are inadequate at reduced temperatures. ${ }^{40}$ For compounds that do not have a high oxygen affinity, such as the $\mathrm{Sr}_{n+1} \mathrm{Cu}_{n} \mathrm{O}_{2 n+1}$ films studied in this work, this could lead to a significant oxygen deficiency in the as grown films, which can be overcome only through high temperature processing. To the extent that the layer-by-layer growth mechanism remains intact, it would be interesting to study the effect of higher oxidation rates at low epitaxial growth temperatures for the synthesis of superconducting derivatives of the IL compound.

\section{ACKNOWLEDGEMENTS}

This research has been supported by the Japan Society for the Promotion of Science, the Yamada Science Foundation, the Laboratory Directed Research and Development Program of the Oak Ridge National Laboratory, and the Division of Materials Sciences, U. S. Department of Energy under contract No. DE-AC05-96OR22464 with Lockheed Martin Energy Research Corp.

\section{REFERENCES}

1. M. Takano, Y. Takeda, H. Okada, M. Miyamoto, and T. Kusaka, Physica C 159, 375 (1989).

2. M. Takano, M. Azuma, Z. Hiroi, Y. Bando, and Y. Takeda, Physica C 176, 441 (1991).

3. M. G. Smith, A. Manthiram, J. Zhao, J. B. Goodenough, and J. T. Markert, Nature 351, 549 (1991).

4. A brief overview is given by: Z. Hiroi and M. Takano, Physica C 235-240, 29 (1994).

5. J. B. Goodenough and A. Manthiram, J. Solid State Chem. 88, 115 (1990).

6. X. Li, M. Kanai, T. Kawai, and S. Kawai, Japan. J. Appl. Phys. 31, L217 (1992).

7. H. Adachi, T. Satoh, Y. Ichikawa, and K. Wasa, Physica C 196, 14 (1992).

8. C. Niu and C. M. Lieber, J. Am. Chem. Soc. 115, 139 (1993).

9. D. P. Norton, B. C. Chakoumakos, J. D. Budai, and D. H. Lowndes, Appl. Phys. Lett. 62, 1679 (1993).

10. A. Gupta, B. W. Hussey, T. M. shaw, A. M. Guloy, M. Y. Chern, R. F. Saraf, and B. A. Scott, J. Sol. State Chem. 112, 113 (1994).

11. M. Yoshimoto, H. Nagata, J. Gong, H. Ohkubo, and H. Koinuma, Physica C 185-189, 2085 (1991).

12. R. Feenstra, X. Li, M. Kanai, T. Kawai, S. Kawai, J. D. Budai, E. C. Jones, Y. R. Sun, J. R. Thompson, S. J. Pennycook, and D. K. Christen, Physica C 224, 300 (1994). 
13. R. Feenstra, J. D. Budai, D. K. Christen, and T. Kawai, Appl. Phys. Lett. 66, 2283 (1995).

14. T. Siegrist, S. M. Zahurak, D. W. Murphy, and R. S. Roth, Nature 324, 231 (1988).

15. G. Er, S. Kikkawa, F. Kanamaru, Y. Mitamoto, S. Tanaka, M. Sera, M. Sato, Z. Hiroi, M. Takano, and Y. Bando, Physica C 196, 271 (1992); N. Ikeda, Z. Hiroi, M. Azuma, M. Takano, Y. Bando, and Y. Takeda, Physica C 210, 367 (1993).

16. D. P. Norton, B. C. Chakoumakos, J. D. Budai, D. H. Lowndes, B. C. Sales, J. R. Thompson, and D. K. Christen, Science 265, 2074 (1994).

17. M. Kawasaki, K. Takahashi, T. Maeda, R. Tsuchiya, M. Shinohara, O. Ishiyama, T. Yonezawa, M. Yoshimoto, and H. Koinuma, Science 266, 1541 (1994).

18. E. C. Jones, D. K. Christen, J. R. Thompson, R. Feenstra, S. Zhu, D. H. Lowndes, J. M. Phillips, M. P. Siegal, and J. D. Budai, Phys. Rev. B 47, 8986 (1993).

19. E. C. Jones, D. P. Norton, B. C. Sales, D. H. Lowndes, and R. Feenstra, Phys. Rev. B 52, R743 (1995).

20. D. P. Norton, B. C. Chakoumakos, E. Jones, D. K. Christen, and D. H. Lowndes, Physica C 217, 146 (1993).

21. M. Azuma, Z. Hiroi, M. Takano, Y. Bando, and Y. Takeda, Nature 356, 775 (1992); Z. Hiroi, M. Azuma, M. Takano, Y. Takeda, Physica C 208, 286 (1993).

22. S. Adachi, H. Yamauchi, S. Tanaka, and N. Mori, Physica C 212, 164 (1993).

23. Z. Liu, T. Hanada, R. Sekina, M. Kawai, and H. Koinuma, Appl. Phys. Lett. 65, 1717 (1994).

24. I. Bozovic and J. N. Eckstein, MRS Bulletin, Vol. 20, (Materials Research Society, Pittsburgh PA, 1995), p. 32.

25. S. J. Pennycook, N. D. Browning, and M. F. Chisholm, in "Studies of High Temperature Superconductors," Vol. 15, Ed. A. Narlikar (Nova Science Publishers, Commack NY, 1995), p. 195.

26. H. Zhang, Y. Y. Wang, H. Zhang, V. P. Dravid, L. D. Marks, P. D. Han, D. A. Payne, P. G. Radaelli, and J. D. Jorgensen, Nature 370, 352 (1994).

27. Y. Shimakawa, J. D. Jorgensen, J. F. Mitchell, B. A. Hunter, H. Shaked, D. G. Hinks, R. L. Hitterman, Z. Hiroi, and M. Takano, Physica C 228, 73 (1994).

28. N. Sugii, M. Ichikawa, K. Hayashi, K. Kubo, K. Yamamoto, and H. Yamauchi, Physica C 213, 345 (1993).

29. J. G. Wen, H. Yakabe, A. Kume, Y. Shiohara, N. Koshiozuka, and S. Tanaka, Physcia C 228, 279 (1994).

30. H. Müller-Buschbaum, Angew. Chem. Int. Ed. Engl. 16, 674 (1977).

31. Z. Hiroi, M. Azuma, M. Takano, and Y. Bando, J. Sol. State Chem. 95, 230 (1991).

32. T. Matsumoto, H. Tanaka, T. Kawai, and S. Kawai, Surface Sci. Lett. 278, L153 (1992).

33. K. Koguchi, T. Matsumoto, T. Kawai, and S. Kawai, Japan. J. Appl. Phys. 33, L514 (1994).

34. K. Koguchi, T. Matsumoto, and T. Kawai, Science 267, 71 (1995).

35. K. Koguchi, T. Matsumoto, and T. Kawai, to appear in Physica C (1996).

36. S. Adachi, H. Yamauchi, S. Tanaka, and N. Mori, Physica C 208, 226 (1993).

37. Z. Hiroi, M. Takano, M. Azuma, and Y. Takeda, Nature 364, 315 (1993).

38. In the case of an endothermic rather than exothermic enthalpy of solution, the oxygen content may increase rather than decrease with increasing temperature.

39. M. A. Alario-Franco, C. Chaillout, J. J. Capponi, J.-L. Tholence, and B. Souletie, Physica C 222, 52 (1994); X.-J. Wu, S. Adachi, C.-Q. Jin, H. Yamauchi, and S. Tanaka, Physica C 223, 243 (1994); H. Ihara, K. Tokiwa, H. Ozawa, M. Hirabayashi, A. Negishi, H. Matuhata, and Y. S. Song, Japan. J. Appl. Phys. 33, L503 (1994).

40. J. A. Kittl, W. L. Johnson, and C. W. Nieh, J. Mater. Res. 7, 2003 (1992). 